\title{
Co-Supplementation of Flos Sophorae Extract with Tremella fuciformis Polysaccharides Improves Physicochemical, Textural, Rheological, and Antioxidant Properties of Low-Fat Yogurts
}

\author{
Renyong Tang, ${ }^{1,2}$ Yurong Lu, ${ }^{1}$ Caiyun Hou, ${ }^{1}$ Jiaxuan Peng, ${ }^{1}$ Wei Wang, ${ }^{2}$ and \\ Xiulan Guo (iD) \\ ${ }^{1}$ School of Pharmacy and Biological Engineering, Chengdu University, Chengdu, Sichuan, China \\ ${ }^{2}$ Sichuan Key Laboratory of Meat Processing, Chengdu University, Chengdu, Sichuan, China \\ Correspondence should be addressed to Xiulan Guo; guoxiulan@cdu.edu.cn
}

Received 3 March 2020; Revised 29 May 2020; Accepted 3 June 2020; Published 22 June 2020

Academic Editor: Flora V. Romeo

Copyright (C) 2020 Renyong Tang et al. This is an open access article distributed under the Creative Commons Attribution License, which permits unrestricted use, distribution, and reproduction in any medium, provided the original work is properly cited.

\begin{abstract}
Flos Sophorae extract (FSE) with abundant rutin and Tremella fuciformis polysaccharides (TP) could act as novel natural improvers of low-fat yogurt due to their health benefits and properties of interacting with proteins. This study attempted to understand how FSE or its combination with TP influences physicochemical, textural, and antioxidant properties of low-fat yogurts. The results indicated that the low concentrations of FSE (1.2 or 2.4\%) increased the antioxidant activity, prompted the S. thermophilus growth, water holding capacity (WHC), and textural and sensory properties, and shortened the fermentation time, but reduced the rheological properties of yogurts compared with the control. Co-supplementing appropriate TP with the optimum FSE concentration (2.4\%) improved the rheological properties, and further enhanced the S. thermophilus growth, WHC, textural and antioxidant properties, and sensory scores of yogurts compared with the $2.4 \%$ FSE group, with the best effects at $0.4 \mathrm{mg} / \mathrm{mL}$ TP. Taken together, co-supplementation of polyphenols extract FSE with Tremella fuciformis polysaccharides may be an available strategy to optimize health-promoting properties and overcome defects of low-fat yogurts.
\end{abstract}

\section{Introduction}

Natural polyphenols have drawn attention due to their health-promoting properties, such as antioxidant and antiseptic actions [1], and they could even prevent chronic diseases [2]. Supplementation of probiotic yogurt with polyphenols can be a great alternative for optimizing benefits of yogurt and polyphenols intakes. Grape seed extract and green tea powder were successfully employed for the production of polyphenol fortified yogurts $[3,4]$.

Polyphenols may interact covalently or noncovalently with proteins and therefore modify the physicochemical and textural properties of yogurt. Low-fat yogurts with Pleurotus ostreatus aqueous or Gnaphalium affine extract, containing phenolic compounds, exhibited lower syneresis and more adhesiveness, springiness, and cohesiveness than of control $[5,6]$. Addition of $0.02 \%$ green tea powder reduced the syneresis rate and promoted the strength of the casein network [4]. However, $2 \%$ green tea powder or $1.5-3 \mathrm{~g} / \mathrm{L}$ of grape extract increased the syneresis and reduced the consistencies or gel strength of yogurts $[2,4]$, and these results could be due to excessive polyphenols interactions with milk protein, which can induce crosslinking and network bonds destabilization [7].

Polyphenols could also bind to polysaccharides, while polysaccharides could bind to proteins and inhibit the interactions of proteins and polyphenols [8-10]. Apple pectin could form complexes with whey proteins, which reduce the particle size and charge of the coacervates containing polyphenols and inhibit the protein precipitation with polyphenols [9]. Moreover, soluble polysaccharides, such as pectin, inulin, and guar gum are used as additive in yogurts as thickeners, stabilizer, gelling agents, syneresis control, and prebiotic [11-13]. Therefore, we could assess whether co-supplementation of polyphenols with polysaccharides improves the quality of low-fat yogurts. 
Tremella fuciformis polysaccharides are extracted from one of the longevity tonics, Tremella fuciformis, and display multiple health benefits such as immunomodulation and antitumor activities [14, 15]. Furthermore, Tremella fuciformis polysaccharides show antioxidant and remarkable water retaining properties [16]. So, it can be used as one of the best natural hydrating ingredients and functional bioactive components applied to food.

Flos Sophorae (FS) is a natural source of polyphenols with more than $20 \%$ rutin $[17,18]$ and used as a folk vegetable and traditional Chinese herbal medicine. FS addition enhanced the antioxidant state of Chinese sausages [18], and rutin supplementation ameliorated the gut dysbiosis and inflammatory status induced by high-fat diet [19]. However, few studies have reported about the application of the Flos Sophorae or its extract in yogurt products. Thus, the present study was to evaluate the potential of FS extract and its combination with Tremella fuciformis polysaccharides to enhance the quality of low-fat yogurts.

\section{Material and Methods}

2.1. Materials. The dry Tremella fuciformis and Flos Sophorae were purchased from the local stores in Chengdu. The commercial freeze-dried culture (Beijing Chuanxiu Ltd., Beijing, China), consisting of Lactobacillus bulgaricus and Streptococcus thermophilus, and skim milk powder (Anchor, New Zealand), containing $0.9 \%$ lipids, $32.9 \%$ protein, and $54.5 \%$ lactose, were used for the yogurt production.

2.2. Extraction of Flos Sophorae. Flos Sophorae was milled with a grinder to get its powder, then $10 \mathrm{~g}$ FS powder was mixed with $100 \mathrm{~mL}$ distilled water, and the suspension was extracted under ultrasonication for $30 \mathrm{~min}$ at $100 \mathrm{~W}$ and $100^{\circ} \mathrm{C}$ and subsequently centrifuged at $2000 \mathrm{rpm}$ for $10 \mathrm{~min}$. The supernatant was the crude extract of Flos Sophorae (FSE), collected and stored at $-20^{\circ} \mathrm{C}$ before use. Chemical analysis indicated FSE contained rutin $18.6 \pm 0.00 \mathrm{mg} / \mathrm{mL}$, total phenolics $20.8 \pm 0.12 \mathrm{mg}$ rutin equivalent $/ \mathrm{mL}$, and crude protein $59.2 \pm 0.5 \mathrm{mg} / \mathrm{mL}$.

\subsection{Extraction and Determination of Tremella fuciformis} Polysaccharides. Tremella fuciformis polysaccharides (TP) were extracted according to Chen [14] with some modifications. In brief, $10 \mathrm{~g}$ dried Tremella fuciformis was triturated and then suspended and boiled in $1000 \mathrm{~mL}$ distilled water for $4.5 \mathrm{~h}$ at $100^{\circ} \mathrm{C}$. After filtration, the filtrate was concentrated in a rotary evaporator, and subsequently protein was removed by the Sevag method. Then, the polysaccharides in the solution were precipitated with three volumes of absolute ethanol for $24 \mathrm{~h}$ at $4^{\circ} \mathrm{C}$, collected by centrifugation, and finally lyophilized. The concentration of polysaccharides was determined by the phenol-sulfuric acid method.

2.4. Set Yogurt Production. Yogurt was formulated to contain $12.5 \%(\mathrm{w} / \mathrm{w})$ skim milk solids, $4 \%$ sucrose $(\mathrm{w} / \mathrm{v})$, and various concentrations of addition (FSE or its combination with TP solution) by replacing part of the water. FSE $(0,1.2$, $2.4,3.6,4.8$, and $6.0 \%, \mathrm{v} / \mathrm{v}$ ) was added to produce Con, FSE1.2, FSE2.4, FSE3.6, FSE4.8, and FSE6.0 yogurts (total phenolics were $0,0.25,0.50,0.75,1.00$, and $1.25 \mathrm{mg}$ rutin equivalent $/ \mathrm{mL}$ milk), respectively. After comparing the different FSE yogurts, the different concentrations of TP ( 0 , $0.2,0.4,0.6$, and $0.8 \mathrm{mg} / \mathrm{mL}$ milk) were added on the base of the optimal FSE content to process FSE2.4, FSE-TP0.2, FSETP0.4, FSE-TP0.6, and FSE-TP0.8 yogurts, respectively. TP was dissolved by boiled water at 1:100 (w/v), and then proportional TP solution was mixed with the milk solution to the required concentration. All yogurt milks were heated at $90^{\circ} \mathrm{C}$ for $10 \mathrm{~min}$ and inoculated with $0.1 \%(\mathrm{w} / \mathrm{v}) \mathrm{com}-$ mercial freeze-dried culture after cooling to $44 \pm 1^{\circ} \mathrm{C}$. The prepared milks were aseptically distributed into $100 \mathrm{~mL}$ glass bottles and $50 \mathrm{~mL}$ the centrifuge tubes and then sealed and incubated at $42^{\circ} \mathrm{C}$ until yogurt curded. Then, the fermentation time was recorded, and they were stored at $4^{\circ} \mathrm{C}$. The antioxidant activity and physicochemical, textural, rheological, sensory, and microbiological parameters were evaluated during days $1-3$ of storage at $4^{\circ} \mathrm{C}$. Three replicates were performed, and measurements were made in triplicates.

2.5. Physicochemical Analysis. The physicochemical properties of FSE and yogurts were determined in triplicates as follows: the amount of protein was determined by the microKjeldahl method; the concentration of total polyphenols was quantified using the Folin-Ciocalteu method. Rutin contents in yogurt and FSE samples were measured according to Tang et al. [18].

Yogurt $\mathrm{pH}$ was measured with a $\mathrm{pH}$ meter (Leici PHS$3 \mathrm{E}$, Shanghai, China) fitted with a combined glass electrode. The Dornic acidity was determined according to Arioui et al. [20], and the results were expressed as degree Dornic. Additionally, water holding capacity (WHC) was determined according to the method of Abbasi et al. [21] with some modifications. Briefly, the yogurt fermented in the centrifuge tube was centrifuged at $2000 \mathrm{rpm}$ for $10 \mathrm{~min}$ at $4^{\circ} \mathrm{C}$. The supernatant was removed, and the precipitate was weighed. The WHC value was calculated as follows:

$$
\frac{\text { precipitate weight }}{\text { initial sample weight }} \times 100 \text {. }
$$

2.6. Microbiological Analysis. Yogurt was fermented by $L$. bulgaricus and $S$. thermophilus, and their numbers were counted on MRS and M17 media and incubated under anaerobic conditions at $37^{\circ} \mathrm{C}$ for $72 \mathrm{~h}$. Colony counts were expressed as $\log \mathrm{CFU} / \mathrm{g}$. All of the tests were repeated in triplicates.

2.7. Textural Analysis. The textural properties of the yogurt samples were evaluated by a penetration test using a TA.XT2 Texture Analyzer (Stable Micro Systems, UK) with a P 0.5 probe, according to the methods of Guo et al. [22]. Briefly, all samples were kept at $4^{\circ} \mathrm{C}-6^{\circ} \mathrm{C}$ and the probe penetrated to 
$20 \mathrm{~mm}$. The speed of the probe was $1.0,2.0$, and $2.0 \mathrm{~mm} / \mathrm{s}$ during the pretest, penetration, and withdrawal, respectively. The maximum force represented the yogurts' firmness at the end of the $20 \mathrm{~mm}$ penetration [23], and the adhesiveness was defined as the negative area [24]. The textural properties of all samples were measured in triplicates.

2.8. Rheological Aanalysis. Rheological properties of the yogurts were characterized using the rheometer (Discovery Hybrid Rheometer, TA Instruments, USA) equipped with a cone and plate geometry $\left(50 \mathrm{~mm}\right.$ diameter, $1^{\circ}$ angle and $55 \mu \mathrm{m}$ gap). Yogurt samples $(\sim 3.0 \mathrm{~g})$ were transferred carefully to the platform of the rheometer and equilibrated at $10^{\circ} \mathrm{C}$ for $30 \mathrm{~min}$. A displacement of $0.002 \mathrm{rad}$ was chosen for the frequency sweep to be tested at $0.1-10 \mathrm{rad} / \mathrm{s}$ (in $\log$ progression with 10 points per decade) at a constant strain of $0.1 \%$ and a constant temperature of $10^{\circ} \mathrm{C}$. The storage modulus $\left(G^{\prime}\right)$, loss modulus $\left(G^{\prime \prime}\right)$, loss tangent $\left(\tan \delta=G^{\prime \prime}\right.$ / $G^{\prime}$ ), and difference between $G^{\prime}$ and $G^{\prime \prime}$ were reported.

2.9. Radical Scavenging Activity Analysis. The DPPH radical scavenging activity was determined as described by Tang et al. [18] with slight modifications. Briefly, the lyophilized samples ( $3 \mathrm{~g}$ ) were extracted with $25 \mathrm{~mL} 60 \%$ ethanol by ultrasonication for $1 \mathrm{~h}$ at $100 \mathrm{~W}$ and $50^{\circ} \mathrm{C}$ and centrifuged at $4000 \mathrm{rpm}$ for $5 \mathrm{~min}$. Subsequently, $2 \mathrm{~mL}$ supernatant was mixed with $2 \mathrm{~mL}$ of $0.2 \mathrm{mM}$ DPPH solution in ethanol, and absorbance was detected at $517 \mathrm{~nm}$ after $30 \mathrm{~min}$ of reaction time in the dark at room temperature. All samples were detected in triplicates.

2.10. Sensory Evaluation. Sensory evaluation was accomplished at the second day of the storage, and the yogurt samples were served in white plastic pots labeled in sensory booths with standard lighting. Ten trained panelists were asked to describe a number of properties of the produced yogurts: color and appearance, texture, flavor, taste, and overall acceptability, and a 9-point hedonic scale (1 point: extremely dislike; 9 points: extremely like) was used to determine these four properties.

2.11. Statistical Analysis. The results were expressed as mean \pm SD. Data analysis was performed using SPSS 17.0 (SPSS Inc., Chicago, Il, USA). Statistically significant differences between groups were determined by an analysis of variance (ANOVA) followed by the post hoc test. Statistical significance was set as $p<0.05$.

\section{Results and Discussion}

3.1. Supplementing of FSE on the Antioxidant Activity and Microbial Counts of Yogurts. FS is considered as a natural source of polyphenols, especially of rutin, and exerted powerful antioxidant capacity $[17,18]$. Supplementation of FSE increased the rutin content in yogurts: $0.21,0.43,0.64$, 0.85 , and $1.08 \mathrm{mg} / \mathrm{mL}$ in FSE1.2, FSE2.4, FSE3.6, FSE4.8, and FSE6.0 yogurts, respectively. Correspondingly, all yogurts with FSE exhibited significantly higher $(p<0.05)$ DPPH radical scavenging activity than Con, the antioxidant activity of yogurts increased with an increase in the FSE concentration (Figure 1(a)), and 2.4\% FSE concentration was an inflection point with increased the DPPH scavenging activity by 2.25 times $(p<0.01)$ compared with that of Con.

The $\mathrm{pH}$ and Dornic acidity values were similar among groups (Figure 1(b)), but there were significant differences in the fermentation time (Figure 1(c)); comparing with Con, it was shortened $p<0.05$ by low concentrations of FSE $(1.2-2.4 \%)$, while it was extended when FSE content reached to $6.0 \%(p<0.05)$. The alteration of fermentation time was closely related to the changing number of microorganisms (Figure 1(d)). The addition of FSE promoted (Figure 1(d), $p<0.01$ ) the growth of $S$. thermophilus in all FSE treatments, with the highest number of S. thermophilus at FSE 1.2\%, while it reduced $(p<0.05)$ the number of L. bulgaricus when the concentration was more than $2.4 \%$, which was consistent with results of fermentation time. Similarly, addition of Fuzhuan brick-tea improved the total numbers of $S$. thermophilus in yogurt [25], and natural polyphenols exhibit differential effects on different bacteria [26].

3.2. Supplementation of FSE on Water Holding Capacity and Textural and Rheological Properties of Yogurts. Low-fat or no-fat yogurts are known to have certain problems such as poor texture, more syneresis, and lower water holding capacity (WHC). The whey separation or syneresis could be reduced by increasing the total solids content of milk (especially protein) or adding appropriate natural stabilizers and polyphenols $[5,12,13,27]$.

In this study, supplementation of FSE improved the WHC $(p<0.05)$ from concentration $1.2 \%$ to $4.8 \%$, with the highest WHC at $2.4 \%(92.65 \%, p<0.01)$ compared with that of Con (Figure 2(a)). These data confirmed that supplementing appropriate polyphenols could improve WHC. Polyphenols have a significant affinity for proteins that leads to the formation of soluble complexes by multiple weak interactions (mainly hydrophobic) and hydrogen bonding [5]. Moreover, the enhanced WHC could be relative with increased protein content in FSE yogurts; addition of $1.2-4.8 \%$ FSE resulted in a $1.3-4.7 \%$ higher percentage of protein compared with the control in this study. The proteins had the ability to entrap water in yogurt gel structure, and the manufacturers try to reduce whey separation by increasing the protein content of yogurt [27].

Textural properties, firmness, and adhesiveness are shown in Figure 2(b). The FSE supplementation at $1.2 \%$ increased the firmness and adhesiveness $(p<0.05)$ but reduced them up to $3.6 \%(p<0.05)$ compared with Con (Figure 2(b)). Similar results were found in other studies that skim yogurts with plant extracts containing phenolic compounds exhibited more adhesiveness but lower firmness $[5,6]$. Exceeded FSE reduced the textural parameters (Figure 2(b)), which could be attributed to increased water and excess polyphenols concentrations in the gel system $[4,5]$. Similarly, there were the lower values of $G^{\prime}$ and $G^{\prime \prime}$ for FSE2.4 as compared with Con (Figure 3), which could be due 

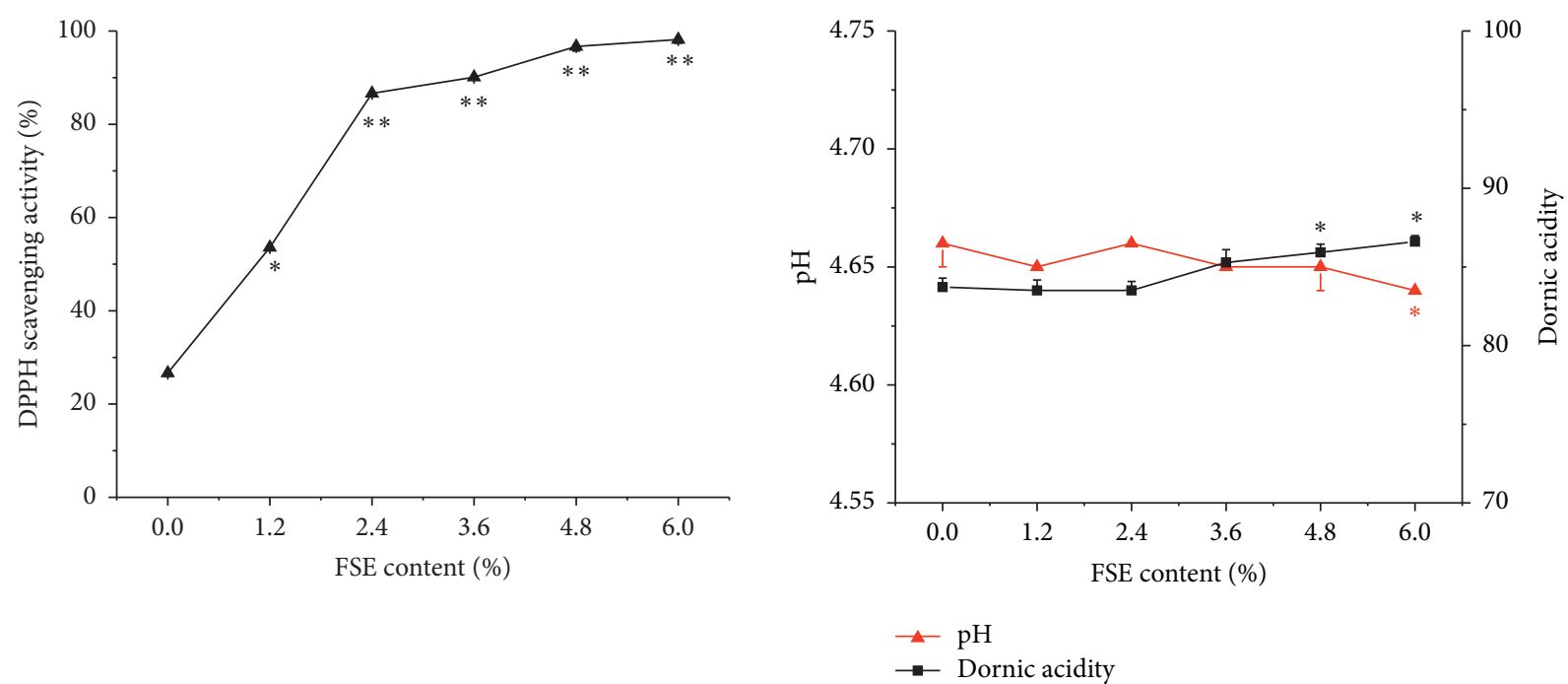

(a)

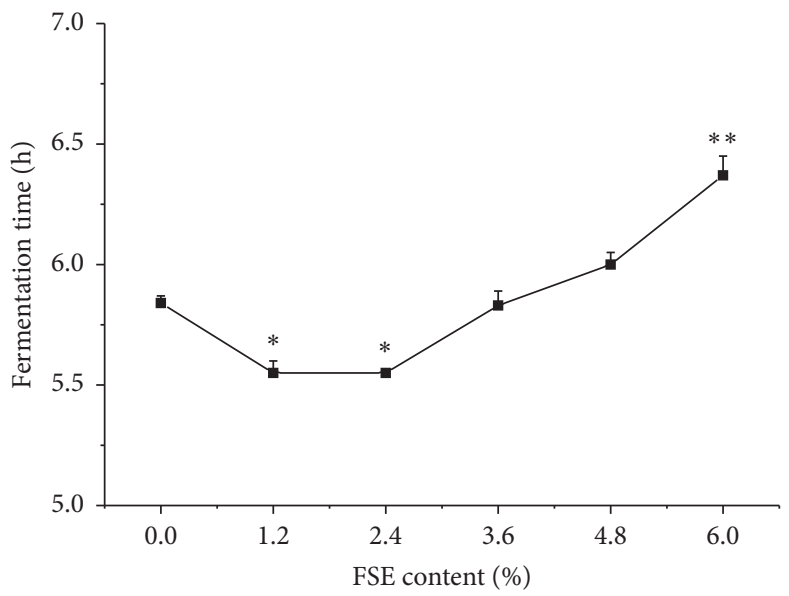

(b)

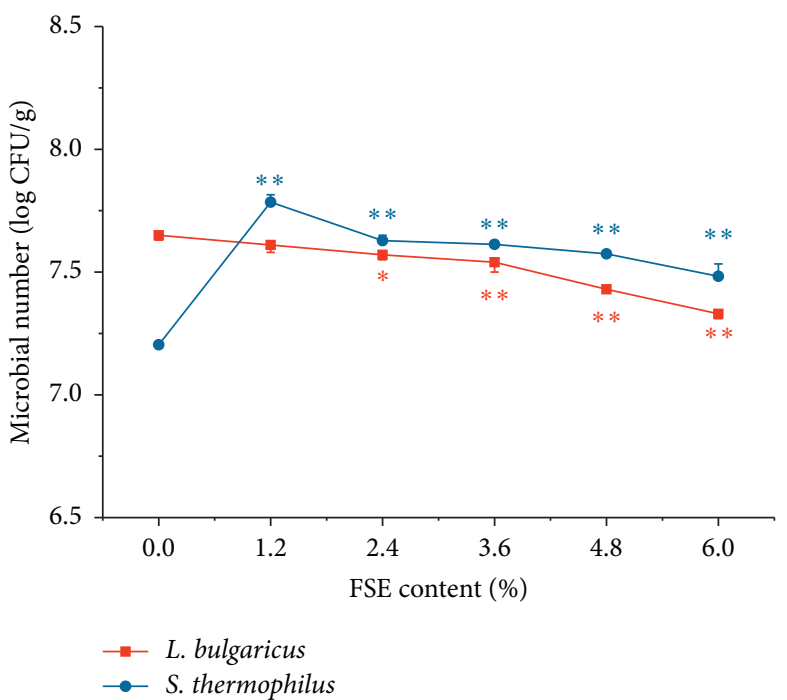

(c)

(d)

FIGURE 1: Effect of supplementing different concentrations of FSE on the antioxidant activity, physicochemical profiles, and microbial counts of yogurts: (a) DPPH scavenging capacity; (b) pH and Dornic acidity; (c) fermentation time; (d) quantities of L. bulgaricus and S. thermophilus. * Significant difference at $p<0.05 ;{ }^{* *}$ significant difference at $p<0.01$ compared with Con. FSE: Flos Sophorae extract; Con: the yogurt without FSE or TP.

to the reason that addition of polyphenols may induce interactions with the hydrophobic surface of milk protein, which would weaken hydrophobic interactions between the amino acid side chains and then reduce the gel strength of protein network [2]. Consistently, lower rheological properties were observed in yogurts with $2 \%$ green tea powder and $1.5-3.0 \mathrm{~g} / \mathrm{L}$ of grape extract $[2,4]$.

3.3. Supplementation of FSE on the Sensory Characteristics of Yogurts. Low-fat or no-fat yogurt was gummy and had a lack of creaminess and more water on the surface due to the loss of fat $[22,28]$. FSE supplementation at the $2.4 \%$ improved the sensory scores with better texture and less whey loss (Table 1). However, FS showed brown and dark color and a little bitter taste $[1,18]$; the excessive concentration of FSE (more than 3.6\%) resulted in higher whey loss and worse taste and color and reduced the appearance, texture, and taste scores, so their yogurts had lower overall acceptability (Table 1).

From the above data, supplementation of FSE at the low concentration (1.2-2.4\%) promoted the growth of $S$. thermophilus, shortened the fermentation time, and improved water holding capacity and textural properties but reduced rheological properties of low-fat yogurts; while yogurt had the best sensory characteristics and higher antioxidant state at the $2.4 \%$ FSE concentration. Therefore, $2.4 \%$ was considered as the optimal FSE content in this study. 


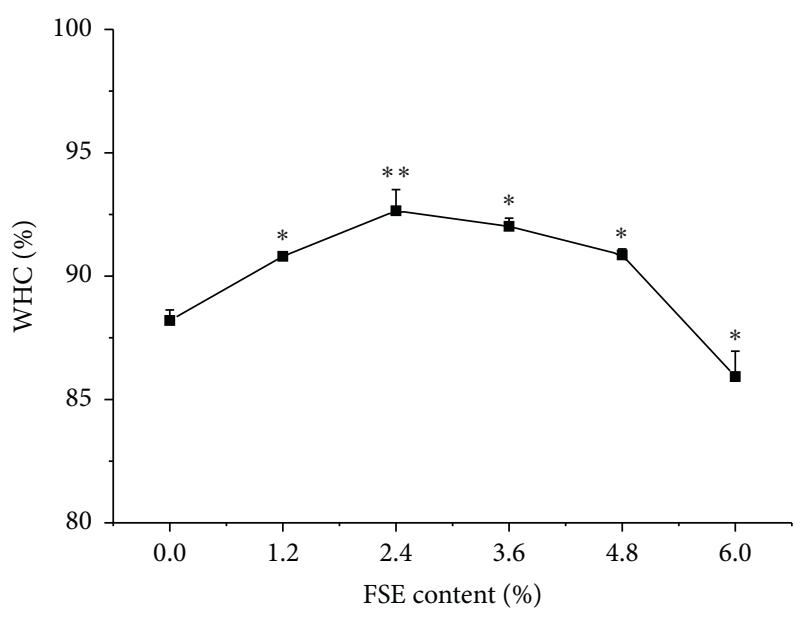

(a)

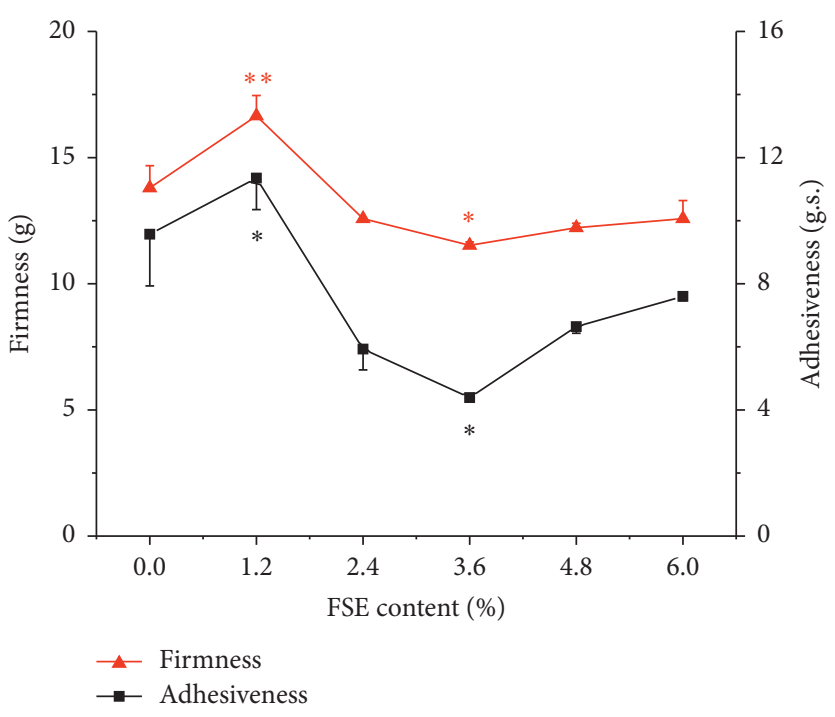

(b)

FIGURE 2: Effect of supplementing different concentrations of FSE on WHC (a) and textural properties (b) of yogurts. * Significant difference at $p<0.05 ;{ }^{* *}$ significant difference at $p<0.01$ compared with Con. FSE: Flos Sophorae extract; WHC: water holding capacity.

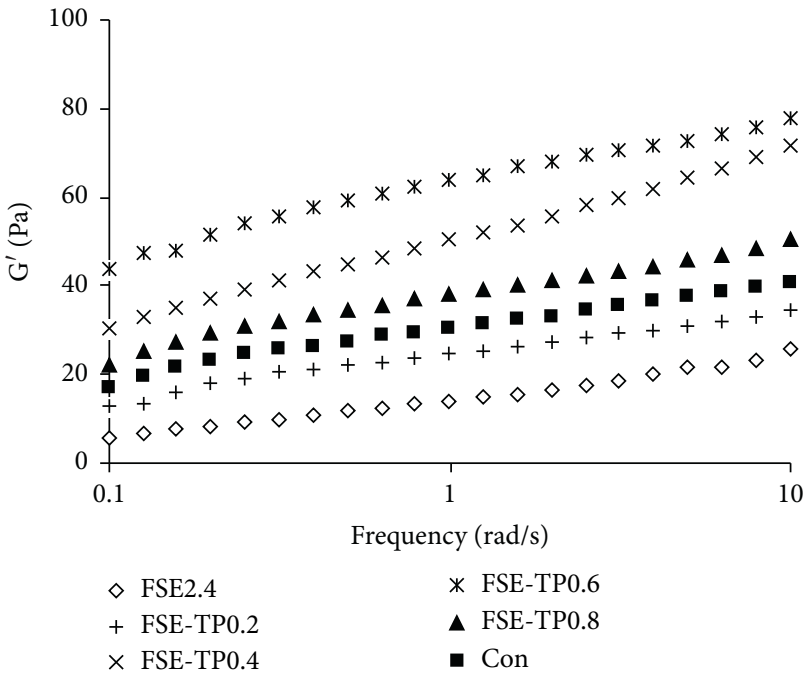

(a)

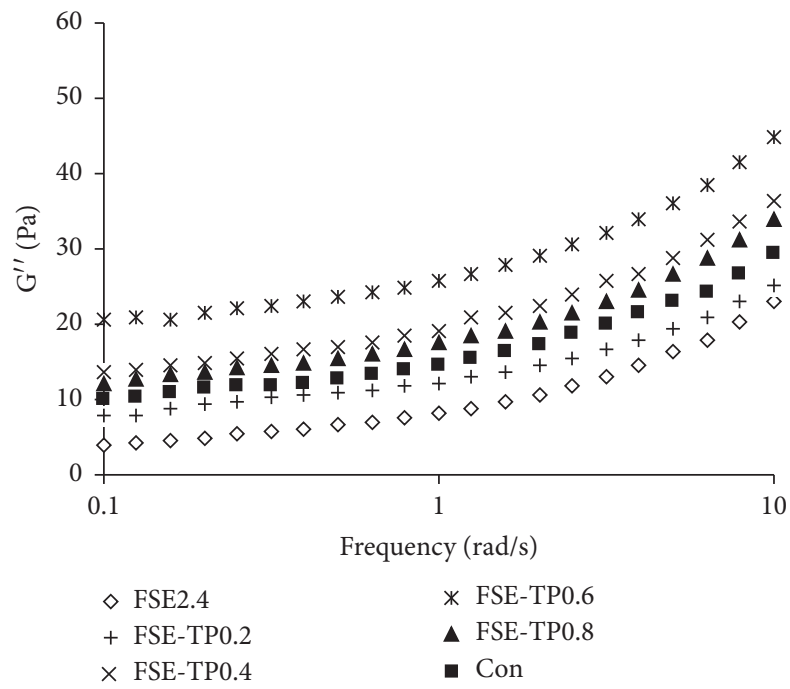

(b)

FIGURE 3: Co-supplementing different contents of TP with 2.4\% FSE affected the rheological properties of yogurts: (a) $G^{\prime}$ (storage modulus) and (b) $G^{\prime \prime}$ (loss modulus). FSE: Flos Sophorae extract; TP: Tremella fuciformis polysaccharides; Con: the yogurt without FSE or TP; based on $2.4 \%$ FSE, the different concentrations of TP $(0,0.2,0.4,0.6$, and $0.8 \mathrm{mg} / \mathrm{mL}$ milk) were added to produce FSE2.4, FSE-TP0.2, FSE-TP0.4, FSE-TP0.6, and FSE-TP0.8 yogurts.

TABLE 1: Effect of supplementing different concentrations of FSE on sensory scores of low-fat yogurts.

\begin{tabular}{lcccc}
\hline Yogurt groups & Flavor and taste & Texture & Color and appearance & Overall acceptability \\
\hline Con & $7.30 \pm 0.28^{\mathrm{a}}$ & $5.94 \pm 0.32^{\mathrm{b}}$ & $6.86 \pm 0.56^{\mathrm{b}}$ & $7.19 \pm 0.26^{\mathrm{c}}$ \\
FSE1.2 & $7.42 \pm 0.33^{\mathrm{a}}$ & $6.60 \pm 0.78^{\mathrm{ab}}$ & $7.96 \pm 0.40^{\mathrm{a}}$ & $7.74 \pm 0.17^{\mathrm{ab}}$ \\
FSE2.4 & $7.55 \pm 0.23^{\mathrm{a}}$ & $6.80 \pm 0.72^{\mathrm{a}}$ & $7.94 \pm 0.62^{\mathrm{a}}$ & $7.84 \pm 0.20^{\mathrm{a}}$ \\
FSE3.6 & $7.57 \pm 0.40^{\mathrm{a}}$ & $6.40 \pm 0.34^{\mathrm{ab}}$ & $6.96 \pm 0.68^{\mathrm{b}}$ & $7.46 \pm 0.27^{\mathrm{b}}$ \\
FSE4.8 & $6.18 \pm 0.48^{\mathrm{b}}$ & $5.86 \pm 0.48^{\mathrm{b}}$ & $6.46 \pm 0.58^{\mathrm{b}}$ & $6.25 \pm 0.23^{\mathrm{d}}$ \\
FSE6.0 & $2.93 \pm 0.35^{\mathrm{c}}$ & $4.54 \pm 0.70^{\mathrm{c}}$ & $5.56 \pm 0.38^{\mathrm{c}}$ & $4.27 \pm 0.28^{\mathrm{e}}$ \\
\hline
\end{tabular}

In each column, the values with different letters indicated significant differences among groups $(p<0.05)$. FSE: Flos Sophorae extract; FSE $(0,1.2,2.4,3.6,4.8$, and $6.0 \%, \mathrm{v} / \mathrm{v}$ ) was added to produce Con, FSE1.2, FSE2.4, FSE3.6, FSE4.8, and FSE6.0 yogurts. 
TABLe 2: The values of $G^{\prime}$ and $G^{\prime \prime}$, differences between $G^{\prime}$ and $G^{\prime \prime}$, and $\tan \delta$ in the linear viscoelastic region.

\begin{tabular}{lcccc}
\hline Yogurt groups & $G^{\prime}(\mathrm{Pa})$ & $G^{\prime \prime}(\mathrm{Pa})$ & $G^{\prime}-G^{\prime \prime}(\mathrm{Pa})$ & \multicolumn{1}{c}{ Tan $\delta$} \\
\hline Con & $27.98 \pm 3.00^{\mathrm{d}}$ & $13.36 \pm 1.67^{\mathrm{c}}$ & $14.62 \pm 1.46^{\mathrm{d}}$ & $0.48 \pm 0.02^{\mathrm{d}}$ \\
FSE2.4 & $11.96 \pm 2.39^{\mathrm{f}}$ & $7.01 \pm 1.53^{\mathrm{e}}$ & $4.94 \pm 0.90^{\mathrm{f}}$ & $0.59 \pm 0.02^{\mathrm{f}}$ \\
FSE-TP0.2 & $22.41 \pm 2.74^{\mathrm{e}}$ & $11.24 \pm 1.40^{\mathrm{d}}$ & $11.17 \pm 1.38^{\mathrm{e}}$ & $0.50 \pm 0.01^{\mathrm{e}}$ \\
FSE-TP0.4 & $45.64 \pm 5.61^{\mathrm{b}}$ & $17.80 \pm 2.22^{\mathrm{b}}$ & $27.85 \pm 3.47^{\mathrm{b}}$ & $0.39 \pm 0.01^{\mathrm{a}}$ \\
FSE-TP0.6 & $59.83 \pm 4.96^{\mathrm{a}}$ & $24.25 \pm 2.06^{\mathrm{a}}$ & $35.57 \pm 2.96^{\mathrm{a}}$ & $0.41 \pm 0.01^{\mathrm{b}}$ \\
FSE-TP0.8 & $35.02 \pm 3.65^{\mathrm{c}}$ & $16.07 \pm 1.87^{\mathrm{b}}$ & $18.95 \pm 1.85^{\mathrm{c}}$ & $0.46 \pm 0.01^{\mathrm{c}}$ \\
\hline
\end{tabular}

FSE: Flos Sophorae extract; TP: Tremella fuciformis polysaccharides; Con: the yogurt without FSE or TP; based on 2.4\% FSE, the different concentrations of TP $\left(0,0.2,0.4,0.6\right.$, and $0.8 \mathrm{mg} / \mathrm{mL}$ milk) were added to produce FSE2.4, FSE-TP0.2, FSE-TP0.4, FSE-TP0.6, and FSE-TP0.8 yogurts; $\tan \delta=G^{\prime \prime} / G^{\prime}$. In each column, the values with different letters indicated significant differences among groups $(p<0.05)$.

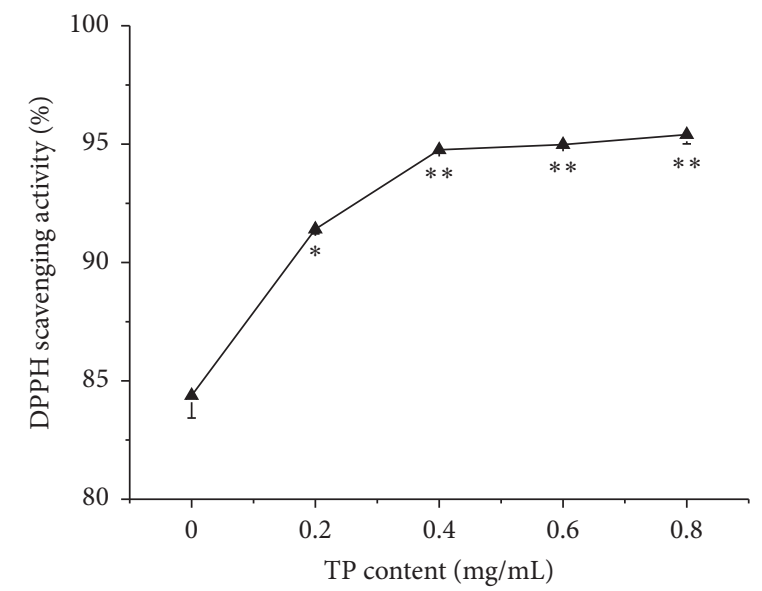

(a)

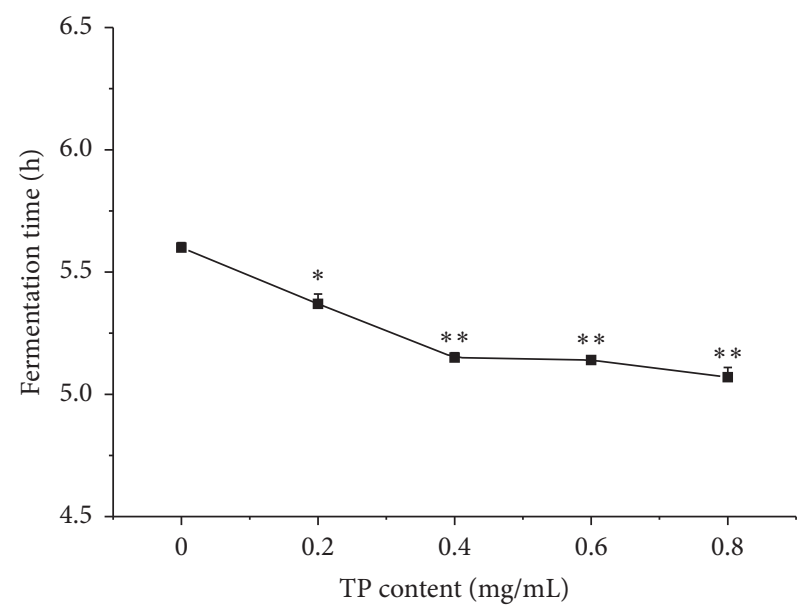

(c)

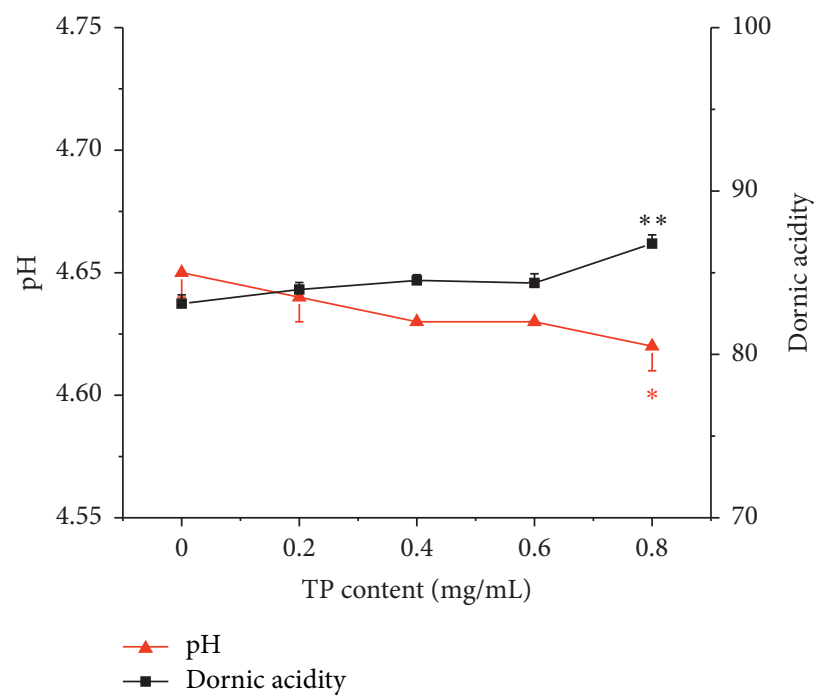

(b)

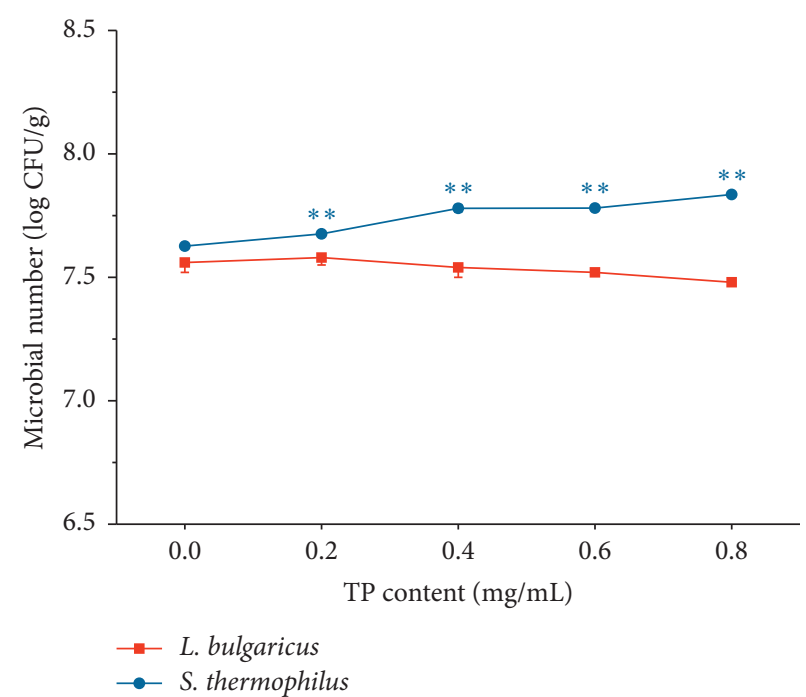

(d)

FIGURE 4: Co-supplementing different contents of TP with 2.4\% FSE influenced on the antioxidant activity, physicochemical profiles, and microbial counts of yogurts: (a) DPPH scavenging capacity; (b) pH and Dornic acidity; (c) fermentation time; (d) quantities of L. bulgaricus and S. thermophilus. " Significant difference at $p<0.05 ;{ }^{* *}$ significant difference at $p<0.01$ compared with FSE2.4. FSE: Flos Sophorae extract; TP: Tremella fuciformis polysaccharides; FSE2.4: the yogurt with $2.4 \%$ FSE. 


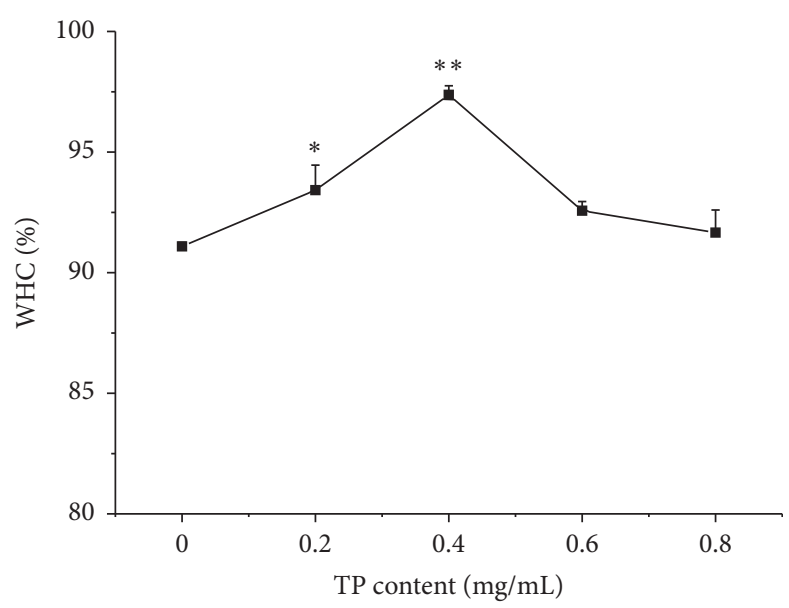

(a)

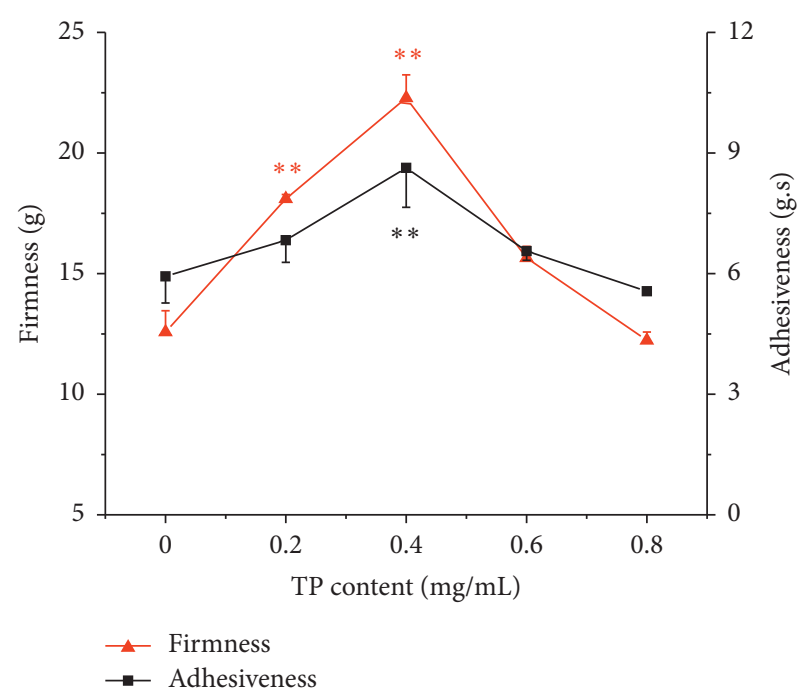

(b)

FIGURE 5: Co-supplementing different contents of TP with 2.4\% FSE influenced on WHC (a) and textural properties (b) of low-fat yogurts. *Significant difference at $p<0.05 ;{ }^{* *}$ significant difference at $p<0.01$ compared with FSE2.4. FSE: Flos Sophorae extract; TP: Tremella fuciformis polysaccharides; WHC: water holding capacity.

3.4. Co-Supplementation of FSE with TP on the Rheological Properties of Yogurts. Rheological properties of yogurts, storage modulus $\left(G^{\prime}\right)$, and loss modulus $\left(G^{\prime \prime}\right)$ are shown in Figure 3 , and the values of loss tangent $(\tan \delta)$ and the difference between $G^{\prime}$ and $G^{\prime \prime}$ in the linear viscoelastic region are given in Table 2. Among all the yogurts, the storage modulus $G^{\prime}$ was greater than loss modulus $G^{\prime \prime}$, which indicated that solid-like properties were predominant in the yogurts. FSE2.4 yogurt had the lowest values for $G^{\prime}$ and $G^{\prime \prime}$ (Figure 3), the lowest difference between $G^{\prime}$ and $G^{\prime \prime}$, and the highest $\tan \delta$ (Table 2). These results suggested that FSE addition could result in yogurt gel weakened, while cosupplementation of Tremella fuciformis polysaccharides with $2.4 \%$ FSE improved the rheological behavior: the $G^{\prime}$ and $G^{\prime \prime}$ values and difference between $G^{\prime}$ and $G^{\prime \prime}$ increased with an increase in TP concentration from 0.2 to $0.6 \mathrm{mg} / \mathrm{mL}$, but the values were reduced at high content of TP $(0.8 \mathrm{mg} / \mathrm{mL})$, and $\tan \delta$ reached the lowest value in the FSE-TP0.4 yogurt. These results could be attributed to the ability of polysaccharides binding to polyphenols, protein, and water molecules, which would inhibit the interactions between polyphenols and proteins and form stable gel network [8-10]. Similarly, polysaccharides such as carrot CWP, gelatin, and apple pomace participated in the overall gel network formation, contributed to a significant increase in both $G^{\prime}$ and $G^{\prime \prime}[23,29]$, elevated the difference between the $G^{\prime}$ and $G^{\prime \prime}$, and improved the rheological behavior to transit from liquid to solid [30]; the incorporation of inulin ( $40 \mathrm{~g} / \mathrm{L})$ and fructans $(60 \mathrm{~g} / \mathrm{L})$ in reduced milk-fat yogurts reduced the $\tan \delta$ values [31].

3.5. Co-Supplementation of FSE with TP on the Antioxidant Activity and Microbial Counts of Yogurts. On the base of the optimum FSE concentration (2.4\%), co-supplementing TP further elevated the DPPH scavenging activity in comparison with the FSE2.4 group, and yogurts had the highest DPPH scavenging activity until TP content reached up to $0.4 \mathrm{mg} / \mathrm{mL}$ (Figure $4(\mathrm{a})$ ), while exceeded TP concentration cannot further increase the antioxidant activity of yogurts. Similarly, Tremella fuciformis polysaccharides exerted a significant scavenging activity on hydroxyl radicals and superoxide radical $[14,16]$.

Polysaccharides have been shown to exert a probiotic role in promoting the growth and reproduction of microorganisms, such as inulin, apple pomace, and polydextrose, resulting in the acceleration of yogurt acid production and shortened fermentation time [28, 29, 32]. Consistently, FSE co-supplementation with TP further promoted the growth of $S$. thermophilus (Figure $4(\mathrm{~d}), p<0.01$ ), and its count was increased while the fermentation time was further decreased (Figure $4(\mathrm{c}), p<0.01$ ) with increase of TP concentration. Fermentation time was shortened closely $30-40 \mathrm{~min}$ by supplementation of TP $0.4-0.6 \mathrm{mg} / \mathrm{mL} \quad(p<0.01)$ when compared with FSE2.4; meanwhile, there was no significant difference in $\mathrm{pH}$ and Dornic acidity (Figure 4(b)).

3.6. Co-Supplementation of FSE with TP on WHC and Textural Properties of Yogurts. Some natural polysaccharides (gelatin, pectin, starches, and gums) were added to ensure appropriate texture and improve the WHC or syneresis $[12,13,23]$. As expected, on the base of $2.4 \%$ FSE, supplementing lower content of TP $(0.2-0.4 \mathrm{mg} / \mathrm{mL})$ further enhanced WHC, with the highest WHC at $0.4 \mathrm{mg} / \mathrm{mL}$ (97.37\%, $p<0.01$ ) compared with FSE2.4 (Figure 5(a)), while exceeded polysaccharides decreased WHC; co-supplementation of lower content of TP with $2.4 \%$ FSE improved the textural properties, with the highest firmness and adhesiveness at TP $0.4 \mathrm{mg} / \mathrm{mL}(p<0.01$, Figure 5(b)). These 
TABLE 3: Effect of co-supplementing different concentrations of TP with the 2.4\% FSE on sensory scores of yogurts.

\begin{tabular}{lcccc}
\hline Yogurt groups & Flavor and taste & Texture & Color and appearance & Overall acceptability \\
\hline FSE2.4 & $7.44 \pm 0.43^{\mathrm{a}}$ & $6.80 \pm 0.28^{\mathrm{a}}$ & $7.60 \pm 0.31^{\mathrm{b}}$ & $7.70 \pm 0.29^{\mathrm{b}}$ \\
FSE-TP0.2 & $7.48 \pm 0.33^{\mathrm{a}}$ & $6.80 \pm 0.28^{\mathrm{a}}$ & $7.84 \pm 0.48^{\mathrm{b}}$ & $7.80 \pm 0.22^{\mathrm{ab}}$ \\
FSE-TP0.4 & $7.44 \pm 0.30^{\mathrm{a}}$ & $7.20 \pm 0.28^{\mathrm{a}}$ & $8.48 \pm 0.36^{\mathrm{a}}$ & $8.06 \pm 0.12^{\mathrm{a}}$ \\
FSE-TP0.6 & $7.44 \pm 0.36^{\mathrm{a}}$ & $6.80 \pm 0.40^{\mathrm{a}}$ & $7.44 \pm 0.53^{\mathrm{b}}$ & $7.60 \pm 0.28^{\mathrm{b}}$ \\
FSE-TP0.8 & $7.20 \pm 0.32^{\mathrm{a}}$ & $5.44 \pm 0.46^{\mathrm{b}}$ & $5.96 \pm 0.46^{\mathrm{c}}$ & $6.74 \pm 0.23^{\mathrm{d}}$ \\
\hline
\end{tabular}

FSE: Flos Sophorae extract; TP: Tremella fuciformis polysaccharides; Con: the yogurt without FSE or TP; based on 2.4\% FSE, the different concentrations of TP $(0,0.2,0.4,0.6$, and $0.8 \mathrm{mg} / \mathrm{mL}$ milk) were added to produce FSE2.4, FSE-TP0.2, FSE-TP0.4, FSE-TP0.6, and FSE-TP0.8 yogurts. In each column, the values with different letters indicated significant differences among groups $(p<0.05)$.

data confirmed that supplementing appropriate polysaccharides could improve WHC and textural parameters. This was similar to the previous reports, which indicated that polysaccharides are easily combined with protein, polyphenols, and water molecules through forming hydrogen bonding, electrostatic forces, and covalent bonds [5, 9], and these could be beneficial to enhance the strength of protein gel network, maintain the water in the network, and reduce the syneresis $[33,34]$.

3.7. Co-Supplementation of FSE with TP on the Sensory Characteristics of Yogurts. On the base of 2.4\% FSE, TP supplementation at $0.4 \mathrm{mg} / \mathrm{mL}$ further improved the appearance scores because it had less whey (Table 3 ). While the higher concentration of TP (more than $0.6 \mathrm{mg} / \mathrm{mL}$ ) resulted in higher whey loss and reduced the appearance and texture scores, they had lower overall acceptability. These results were consistent with the results of WHC and textural properties (Figure 5) and indicated the co-supplementation of appropriate TP with FSE could improve sensory defects of low-fat yogurt. Consistently, adding proper amount of polysaccharides (inulin or Jerusalem artichoke powder) successfully improved the creaminess and reduced the surface water of low-fat yogurts, while excess supplementation resulted in a weaker texture of the yogurt $[22,35]$.

\section{Conclusions}

In summary, the present study demonstrated that supplementation of FSE at the low concentration promoted the growth of S. thermophilus, shortened the fermentation time, and improved antioxidant state, water holding capacity, and textural and sensory properties of low-fat yogurts, but reduced rheological properties of yogurts. The co-supplementation of $2.4 \%$ FSE with low content of TP not only improved rheological properties of yogurts but also further magnified the above effects, with the best effects at $0.4 \mathrm{mg} /$ $\mathrm{mL}$ TP. Therefore, co-supplementing appropriate polyphenol extract FSE and Tremella fuciformis polysaccharides may be a promising potential strategy to improve quality and health-promoting properties of low-fat yogurts.

\section{Data Availability}

The data used to support the findings of this study are available from the corresponding author upon request.

\section{Conflicts of Interest}

The authors declare that there are no conflicts of interest regarding the publication of this paper.

\section{Acknowledgments}

This project was financially supported by the Sichuan Provincial Education Department (project no. 18ZB0136).

\section{References}

[1] X. He, Y. Bai, Z. Zhao et al., "Local and traditional uses, phytochemistry, and pharmacology of sophora japonica L.: a review," Journal of Ethnopharmacology, vol. 187, pp. 160-182, 2016.

[2] D. Felix da Silva, N. N. T. Junior, R. G. Gomes, M. S. dos Santos Pozza, M. Britten, and P. T. Matumoto-Pintro, "Physical, microbiological and rheological properties of probiotic yogurt supplemented with grape extract," Journal of Food Science and Technology, vol. 54, no. 6, pp. 1608-1615, 2017.

[3] V. Chouchouli, N. Kalogeropoulos, S. J. Konteles, E. Karvela, D. P. Makris, and V. T. Karathanos, "Fortification of yoghurts with grape (vitis vinifera) seed extracts," LWT-Food Science and Technology, vol. 53, no. 2, pp. 522-529, 2013.

[4] Ö. Dönmez, B. A. Mogol, and V. Gökmen, "Syneresis and rheological behaviors of set yogurt containing green tea and green coffee powders," Journal of Dairy Science, vol. 100, no. 2, pp. 901-907, 2017.

[5] A. C. Pelaes Vital, P. A. Goto, L. N. Hanai et al., "Microbiological, functional and rheological properties of low fat yogurt supplemented with pleurotus ostreatus aqueous extract," LWT-Food Science and Technology, vol. 64, no. 2, pp. 1028-1035, 2015.

[6] H.-X. Gao, Z.-L. Yu, Q. He, S.-H. Tang, and W.-C. Zeng, “A potentially functional yogurt co-fermentation with gnaphalium affine," LWT-Food Science and Technology, vol. 91, pp. 423-430, 2018.

[7] J. Han, M. Britten, D. St-Gelais et al., "Effect of polyphenolic ingredients on physical characteristics of cheese," Food Research International, vol. 44, no. 1, pp. 494-497, 2011.

[8] S. Soares, N. Mateus, and V. de Freitas, "Carbohydrates inhibit salivary proteins precipitation by condensed tannins," Journal of Agricultural and Food Chemistry, vol. 60, no. 15, pp. 3966-3972, 2012.

[9] C. Thongkaew, M. Gibis, J. Hinrichs, and J. Weiss, "Polyphenol interactions with whey protein isolate and whey protein isolate-pectin coacervates," Food Hydrocolloids, vol. 41, pp. 103-112, 2014. 
[10] C. Le Bourvellec and C. M. G. C. Renard, "Interactions between polyphenols and macromolecules: quantification methods and mechanisms," Critical Reviews in Food Science and Nutrition, vol. 52, no. 3, pp. 213-248, 2012.

[11] F. P. Bruzantin, J. L. P. Daniel, P. P. M. da Silva, and M. H. F. Spoto, "Physicochemical and sensory characteristics of fat-free goat milk yogurt with added stabilizers and skim milk powder fortification," Journal of Dairy Science, vol. 99, no. 5, pp. 3316-3324, 2016.

[12] R. P. d. S. Oliveira, P. Perego, M. N. d. Oliveira, and A. Converti, "Effect of inulin as prebiotic and synbiotic interactions between probiotics to improve fermented milk firmness," Journal of Food Engineering, vol. 107, no. 1, pp. 36-40, 2011.

[13] L. K. Hassan, H. F. Haggag, M. H. ElKalyoubi, M. Abd ELAziz, M. M. El-Sayed, and A. F. Sayed, "Physico-chemical properties of yoghurt containing cress seed mucilage or guar gum," Annals of Agricultural Sciences, vol. 60, no. 1, pp. 21-28, 2015.

[14] B. Chen, "Optimization of extraction of tremella fuciformis polysaccharides and its antioxidant and antitumour activities in vitro," Carbohydrate Polymers, vol. 81, no. 2, pp. 420-424, 2010.

[15] X. Du, J. Zhang, Z. Lv, L. Ye, Y. Yang, and Q. Tang, “Chemical modification of an acidic polysaccharide (TAPA1) from tremella aurantialba and potential biological activities," Food Chemistry, vol. 143, pp. 336-340, 2014.

[16] X. Wang, Z. Zhang, and M. Zhao, "Carboxymethylation of polysaccharides from tremella fuciformis for antioxidant and moisture-preserving activities," International Journal of Biological Macromolecules, vol. 72, pp. 526-530, 2015.

[17] R. Wang, Y. Chang, Z. Tan, and F. Li, "A novel combined process for extracting, separating and recovering flavonoids from flos sophorae immaturus," Separation and Purification Technology, vol. 172, pp. 422-432, 2017.

[18] R. Tang, J. Luo, W. Wang, D. Liu, G. Wang, and X. Guo, "Rutin's natural source flos sophorae as potential antioxidant and improver of fungal community in Chinese sausages," LWT, vol. 101, pp. 435-443, 2019.

[19] X. Guo, R. Tang, S. Yang et al., "Rutin and its combination with inulin attenuate gut dysbiosis, the inflammatory status and endoplasmic reticulum stress in paneth cells of obese mice induced by high-fat diet," Frontiers in Microbiology, vol. 9, p. 2651, 2018.

[20] F. Arioui, D. Ait Saada, and A. Cheriguene, "Physicochemical and sensory quality of yogurt incorporated with pectin from peel of citrus sinensis," Food Science \& Nutrition, vol. 5, no. 2, pp. 358-364, 2016.

[21] H. Abbasi, M. E. Mousavi, M. R. Ehsani et al., "Influence of starter culture type and incubation temperatures on rheology and microstructure of low fat set yoghurt," International Journal of Dairy Technology, vol. 62, no. 4, pp. 549-555, 2009.

[22] X. Guo, Z. Xie, G. Wang, Q. Zou, and R. Tang, "Effect on nutritional, sensory, textural and microbiological properties of low-fat yoghurt supplemented with Jerusalem artichoke powder," International Journal of Dairy Technology, vol. 71, pp. 167-174, 2018.

[23] T. H. McCann, F. Fabre, and L. Day, "Microstructure, rheology and storage stability of low-fat yoghurt structured by carrot cell wall particles," Food Research International, vol. 44, no. 4, pp. 884-892, 2011.

[24] G. D. Kusuma, T. Paseephol, and F. Sherkat, "Prebiotic and rheological effects of Jerusalem artichoke inulin in low-fat yogurt," Australian Journal of Dairy Technology, vol. 64, pp. 159-163, 2009.

[25] D. Liu, "Effect of fuzhuan brick-tea addition on the quality and antioxidant activity of skimmed set-type yoghurt," International Journal of Dairy Technology, vol. 71, pp. 22-33, 2018.

[26] C.-L. Chan, R.-Y. Gan, N. P. Shah, and H. Corke, "Polyphenols from selected dietary spices and medicinal herbs differentially affect common food-borne pathogenic bacteria and lactic acid bacteria," Food Control, vol. 92, pp. 437-443, 2018.

[27] P. Vareltzis, K. Adamopoulos, E. Stavrakakis, A. Stefanakis, and A. M. Goula, "Approaches to minimise yoghurt syneresis in simulated tzatziki sauce preparation," International Journal of Dairy Technology, vol. 69, no. 2, pp. 191-199, 2016.

[28] N. Srisuvor, N. Chinprahast, C. Prakitchaiwattana, and S. Subhimaros, "Effects of inulin and polydextrose on physicochemical and sensory properties of low-fat set yoghurt with probiotic-cultured banana purée," LWT-Food Science and Technology, vol. 51, no. 1, pp. 30-36, 2013.

[29] X. Wang, E. Kristo, and G. LaPointe, "The effect of apple pomace on the texture, rheology and microstructure of set type yogurt," Food Hydrocolloids, vol. 91, pp. 83-91, 2019.

[30] P. Mudgil, B. Jumah, M. Ahmad, F. Hamed, and S. Maqsood, "Rheological, micro-structural and sensorial properties of camel milk yogurt as influenced by gelatin," Lwt, vol. 98, pp. 646-653, 2018.

[31] G. Crispín-Isidro, C. Lobato-Calleros, H. Espinosa-Andrews, J. Alvarez-Ramirez, and E. J. Vernon-Carter, "Effect of inulin and agave fructans addition on the rheological, microstructural and sensory properties of reduced-fat stirred yogurt," LWT-Food Science and Technology, vol. 62, no. 1, pp. 438-444, 2015.

[32] R. P. de Souza Oliveira, P. Perego, M. N. de Oliveira, and A. Converti, "Effect of inulin on the growth and metabolism of a probiotic strain of lactobacillus rhamnosus in co-culture with Streptococcus thermophilus," LWT, vol. 47, no. 2, pp. 358-363, 2012.

[33] S. de Jong and F. van de Velde, "Charge density of polysaccharide controls microstructure and large deformation properties of mixed gels," Food Hydrocolloids, vol. 21, no. 7, pp. 1172-1187, 2007.

[34] R. Fu, J. Li, T. Zhang et al., "Salecan stabilizes the microstructure and improves the rheological performance of yogurt," Food Hydrocolloids, vol. 81, pp. 474-480, 2018.

[35] P. Kip, D. Meyer, and R. H. Jellema, "Inulins improve sensoric and textural properties of low-fat yoghurts," International Dairy Journal, vol. 16, no. 9, pp. 1098-1103, 2006. 